\title{
Interval Valued Fuzzy Soft Sets and Algorithm of IVFSS Applied to the Risk Analysis of Prostate Cancer
}

\author{
Muhammad Naveed \\ Jafar \\ Department of \\ Mathematics \\ Lahore Garrison \\ University \\ Lahore - 54000 Pakistan
}

\author{
Muhammad Riaz \\ Khan \\ Department of \\ Mathematics \\ Lahore Garrison \\ University
}

Lahore - 54000 Pakistan

\author{
Hamid Sultan \\ Department of \\ Mathematics \\ Lahore Garrison \\ University, \\ Lahore - 54000 Pakistan
}

\author{
Naveed Ahmed \\ Department of \\ Mathematics \\ Lahore Garrison \\ University \\ Lahore - 54000 Pakistan
}

\begin{abstract}
In the past two decades, the frequent usage of computers has been observed as it has advanced and has aided us in several fields including medical. In the following study, we deduce an algorithm by deriving results from the correlation of prostate specific antigen (PSA), prostate volume (PV), PSA density, and the age of the already diagnosed prostate cancer patients and patients under consideration. This algorithm helps us develop a calculator that will determine the potential cancer risk and chances of BPH as well.
\end{abstract}

\section{Keywords}

Prostate Cancer, IVFSS, Fuzzy Soft Sets, PSA, Age Factor, $\mathrm{BPH}$

\section{INTRODUCTION}

Several fields corresponding to medical, engineering, economics, etc., require more than ordinary mathematical approach. The numerical model in itself is quite inordinate and a solution cannot be produced without setting appropriate parameters. Various decision-making theories have been devised over time to tackle ambiguous concepts where the numerical methodologies are not sufficient to provide a suitable basis to solve a certain problem in question. By default, we use crisp set theory where a attribute can either exist or it cannot (true or false).

One of the most important milestones in the quest to tackle doubt inducing scenarios is the fuzzy set theory put forward by Zadeh[1]. The usage of FST is quite common in fields corresponding to medical, engineering, economics and other practical applications. The fuzzy set theory has the basis on a membership function assigned within the interval $[0,1]$ which is further briefed in this study. The membership function helps us deduce the membership degree of an element corresponding to a set within which it is located. A fuzzy set can be determined by its membership function. FST has been popularized due to its usage in different scenarios. There exists a hurdle to its diversity in applications however, the deficiency of parameters hurts this theory [2]. Molodtsov [2] introduced a new theory to solve problems which present ambiguity. Molodtsov displayed that his theory aids in several applications such as game theory, Riemann-integration, Perron-integration, etc. Moldotsov's presented theory does not suffer from the same deficiency of parameters as others prior to it do. Soft sets can be portrayed in Boolen-valued information system. Later on, Maji et al. [3] presented a hybrid of the fuzzy sets and soft sets i.e. fuzzy soft set theory. The theory was further developed by Irfan Ali and Shabir [4]. Feng et al. addressed the usage of fuzzy soft sets by introducing the concept of level soft sets of fuzzy soft sets [5]. Feng et al. provided the idea of amalgamating soft sets, fuzzy sets and rough sets. [6]. Noticing the unrealistic standards in FSS, Yang introduced the concept of IVFSS [7]. IVFSST is more flexible in multi-criteria decision-making scenarios and is more helpful when the data set has a significantly large scale.

Prostate cancer is one of the most common cancers among men. It is influenced by various factors such as family cancer history, ethnicity and most importantly the PSA level. PSA testing is the first step in diagnosis for potential patients. [8-10]. But taking PSA levels as the standalone basis for diagnosis is unreliable as high PSA levels could also be an indication of benign prostate hyperplasia (BPH). The precise measures for determining prostate cancer is prostate biopsy. Results of PSA test, rectal examination and transrectal observations helps the doctor decide the necessity of prostate biopsy. [11-13]. Prostate Biopsy, however, can cause complications and comes with a high cost. Hence some patients skip it all together. There have been several studies and research works performed for determining prostate cancer risk and distinguishing it from BPH. FES is a rule-based fuzzy expert system utilizing values of PSA, PV and age of the patient to help a doctor determine the need of prostate biopsy [14]. Keles et al. [15] created a neuro-fuzzy classifier for diagnosis of prostate cancer and BPH. This is important as it helps in differentiating the two since the symptoms can be quite similar. Saritas et al. [16] produced an artificial neural network to be used as prognosis for prostate cancer risk by taking PPSA, tPSA and age data as input. Researchers [25-33] are doing research in soft set theory and use it in decision making problems.

The following study uses FSST and IVFSST to determine the impact factors of age, PSA, PV and PSA density in prostate cancer risk. Using the results from our study, we devise an algorithm for calculating prostate cancer risk. This algorithm when coded, can be used as a prognosis tool.

\section{PREREQUISITES}

Fuzzy Set: Declare a universal set $\mathrm{U}$ and a fuzzy set $\mathrm{X} \subseteq \mathrm{U}$. The set $\mathrm{X}$ will be written as

$$
X=\left\{\left(x, \alpha_{X}(x)\right) \mid x \in U\right\}
$$

Such that

$$
\alpha_{x}: U \rightarrow[0,1]
$$


$\alpha_{X}(x)$ Describes the membership percentage of $x \in X$. A fuzzy set can be compared to crisp sets, soft sets, IVFSs and IVFSSs.

Soft Set: Consider a universal set $\mathrm{U}$ with set of parameters $\mathrm{E}$. Using this combination, we can declare a soft universe (U,E). The power set of $U$ written as $P(U)$ consists of all the subsets of U. A soft set within this universe can be written as $(F, E)$ where

$$
F: E \rightarrow P(U)
$$

Hence soft set is the set of subsets of $P(U)_{\text {subject to certain }}$ parameters.

Fuzzy Soft Set: Similarly we can declare a fuzzy soft set within this universe. Let $\mathrm{I}(\mathrm{U})$ be set of all fuzzy subsets. A fuzzy soft set can be defined as $(\mathrm{F}, \mathrm{E})$ where

$$
F: E \rightarrow I(U)
$$

In fuzzy soft sets, each subset, subject to a parameter, is granted a membership percentage.

Interval-Valued Fuzzy Set: In the same universe, let $\mathrm{X}$ be a IVFS such that

$$
X: U \rightarrow \text { Interval }([0,1])
$$

Interval $([0,1])$ indicates set of all closed sub-intervals and all the IVFSs are contained within P(U). For one such IVFS X,

$(F, E)=\left\{\begin{array}{l}e_{1}=\left\{v_{1}, v_{3}\right\}, e_{2}=\left\{v_{4}\right\}, e_{3}=\left\{v_{2}, v_{5}, v_{7}\right\}, e_{4}=\left\{v_{1}, v_{4}, v_{5}\right\}, e_{5}=\left\{v_{6}, v_{7}\right\} \\ e_{6}=\left\{v_{1}, v_{2}\right\}, e_{7}=\left\{v_{6}\right\}\end{array}\right\}$

$$
\text { consider } \quad x \in U
$$

then

$\alpha_{X}(x)=\left[\alpha_{X(L L)}(x), \alpha_{X(U L)}(x)\right]$

denotes the

membership degree of $x$ in $\mathrm{X}$. $\beta_{X(L L)}(x)_{\text {represents the }}$ lower and $\beta_{X(U L)}(x)$ the upper degree of membership $x$ where $0 \leq \beta_{X(L L)}(x) \leq \beta_{X(U L)}(x) \leq 1$

Interval-Valued Fuzzy Soft Set: Take (F,E) as an IVFSS over the same soft universe then the set of parametric membership functions of the following set can be defined as

$$
\alpha_{(F, E)}=\left\{\alpha_{(F, E)}^{(p)} \mid p \in E\right\}
$$

The functions contained within this set are defined as:-

$$
\alpha_{(F, E)}^{(p)}(x)=t, t \in[0,1]
$$

Example: Suppose you are to purchase a laptop for your office work, home use and/or gaming. You want to ensure that this laptop is both reliable and comes cheap but you don't want to compromise on the other set of features i.e. powerful CPU, GPU, enough storage, sufficient RAM and portability. Let the set of laptops available be $U=\{\mathrm{v} 1, \mathrm{v} 2, \mathrm{v} 3, \mathrm{v} 4, \mathrm{v} 5, \mathrm{v} 6, \mathrm{v} 7\}$ and the set of features(parameters) be $\mathrm{E}=\{\mathrm{e} 1, \mathrm{e} 2, \mathrm{e} 3, \mathrm{e} 4, \mathrm{e} 5, \mathrm{e} 6, \mathrm{e} 7\}$. The soft set $(\mathrm{F}, \mathrm{E})$ displaying the existence of the said features within the vehicles available is as follows:- or

$\mathrm{F}(\mathrm{e} 1)=\{\mathrm{v} 1, \mathrm{v} 3\}$

$\mathrm{F}(\mathrm{e} 2)=\{\mathrm{v} 4\}$
$\mathrm{F}(\mathrm{e} 3)=\{\mathrm{v} 2, \mathrm{v} 5, \mathrm{v} 7\}$

$\mathrm{F}(\mathrm{e} 4)=\{\mathrm{v} 1, \mathrm{v} 4, \mathrm{v} 5\}$

$\mathrm{F}(\mathrm{e} 5)=\{\mathrm{v} 6, \mathrm{v} 7\}$

$\mathrm{F}(\mathrm{e} 6)=\{\mathrm{v} 1, \mathrm{v} 2\}$

$\mathrm{F}(\mathrm{e} 7)=\{\mathrm{v} 6\}$

This can be represented in tabular form or matrix form to provide further clarity of the tradeoff for each vehicle.

$\begin{array}{cccccccc} & e_{1} & e_{2} & e_{3} & e_{4} & e_{5} & e_{6} & e_{7} \\ v_{1} & 1 & 0 & 0 & 1 & 0 & 1 & 0 \\ v_{2} & 0 & 0 & 1 & 0 & 0 & 1 & 0 \\ v_{3} & 1 & 0 & 0 & 0 & 0 & 0 & 0 \\ v_{4} & 0 & 1 & 0 & 1 & 0 & 0 & 0 \\ v_{5} & 0 & 0 & 1 & 1 & 0 & 0 & 0 \\ v_{6} & 0 & 0 & 0 & 0 & 1 & 0 & 1 \\ v_{7} & 0 & 0 & 1 & 0 & 1 & 0 & 0\end{array}$

This certainly proves useful to some extent as we observe the presence and absence of features in the product. However, some features are not completely lost to a product and tend to be present to a certain degree. This is where it is better to use FSS to represent a certain scenario. Suppose a fuzzy soft set $(F, E)$ displays the assumed membership degree of the features in each laptop i.e.

$\mathrm{F}(\mathrm{e} 1)=\{(\mathrm{v} 1,0.7),(\mathrm{v} 2,0.3),(\mathrm{v} 3,0.5),(\mathrm{v} 4,0.4),(\mathrm{v} 6,0.1)\}$

$\mathrm{F}(\mathrm{e} 2)=\{(\mathrm{v} 1,0.3),(\mathrm{v} 4,0.9),(\mathrm{v} 5,0.2),(\mathrm{v} 7,0.4)\}$

$\mathrm{F}(\mathrm{e} 3)=\{(\mathrm{v} 2,0.8),(\mathrm{v} 3,0.3),(\mathrm{v} 4,0.2),(\mathrm{v} 5,0.9),(\mathrm{v} 7,1)\}$

$\mathrm{F}(\mathrm{e} 4)=\{(\mathrm{v} 1,0.6),(\mathrm{v} 3,0.4),(\mathrm{v} 4,0.9),(\mathrm{v} 5,0.7),(\mathrm{v} 7,0.3)\}$

$\mathrm{F}(\mathrm{e} 5)=\{(\mathrm{v} 3,0.2),(\mathrm{v} 6,0.9),(\mathrm{v} 7,0.8)\}$

$\mathrm{F}(\mathrm{e} 6)=\{(\mathrm{v} 1,0.7),(\mathrm{v} 2,0.9)\}$

$\mathrm{F}(\mathrm{e} 7)=\{(\mathrm{v} 2,0.3),(\mathrm{v} 3,0.2),(\mathrm{v} 4,0.4),(\mathrm{v} 6,0.5)\}$

This can be represented in matrix/ tabular form

$\begin{array}{cccccccc} & e_{1} & e_{2} & e_{3} & e_{4} & e_{5} & e_{6} & e_{7} \\ v_{1} & 0.7 & 0.3 & 0 & 0.6 & 0 & 0.7 & 0 \\ v_{2} & 0.3 & 0 & 0.8 & 0 & 0 & 0.9 & 0.3 \\ v_{3} & 0.5 & 0 & 0.3 & 0.4 & 0.2 & 0 & 0.2 \\ v_{4} & 0.4 & 0.9 & 0.2 & 0.9 & 0 & 0 & 0.4 \\ v_{5} & 0 & 0.2 & 0.9 & 0.7 & 0 & 0 & 0 \\ v_{6} & 0.1 & 0 & 0 & 0 & 0.9 & 0 & 0.5 \\ v_{7} & 0 & 0.4 & 1 & 0.3 & 0.8 & 0 & 0\end{array}$

We observe that this increases our options and hence we can put more thought into our needs and wants. Now, although FSS provides us with ideal numbers for each feature, it is not always viable to assign a single value to a feature as one cannot be too sure. In such scenario, IVFSS is more feasible. IVFSS enables us to determine intervals within $[0,1]$ which can be attributed to each feature within our product. Additionally, some features 
can not be evaluated objectively, so we can still determine their value in subjective matters. Let the membership degrees be

$\mathrm{F}(\mathrm{e} 1)=\{(\mathrm{v} 1,[0.4,0.7]),(\mathrm{v} 2,[0,0.3]),(\mathrm{v} 3,[0.3,0.5]),(\mathrm{v} 4,[0.1,0.4])$, $(\mathrm{v} 6,[0,0.1])\}$

$F(e 2)=\{(v 1,[0.1,0.3]),(v 4,[0.6,0.9]),(v 5,[0,0.2]),(v 7,[0.1,0.4])\}$

$\mathrm{F}(\mathrm{e} 3)=\{(\mathrm{v} 2,[0.5,0.8]),(\mathrm{v} 3,[0,0.3]),(\mathrm{v} 4,[0.1,0.2]),(\mathrm{v} 5,[0.5,0.9])$, $(\mathrm{v} 7,[0.7,1])\}$

$e_{1}$

$e_{2}$

$e_{3}$

$e_{4}$

$v_{1} \quad[0.4,0.7]$

$[0.1,0.3]$

0

$[0.3,0.6]$

$v_{2} \quad[0,0.3]$

0

$[0.5,0.8]$

0

$v_{3}[0.3,0.5]$

0

$v_{4}[0.1,0.4]$

$[0.6,0.9]$

$[0,0.3]$

$[0.1,0.4]$

$[0.1,0.2] \quad[0.5,0.9]$

$v_{5} \quad[0,0.1]$

$[0,0.2]$

$[0.5,0.9]$

$[0.4,0.7]$

$v_{6} \quad[0,0.1]$

0

0

0

$v_{7}$

[0.1,0.4]

$[0.7,1]$

$[0.1,0.3]$

Null Soft Set [17]: Consider (F, A), a soft set in U if $\forall a \in A, F(a)=\phi$ then (F, A) is a null soft set.

Absolute Soft Set[17]: Let (F,A) be a soft set over U then if $\forall a \in A, F(a) \in U$ then (F,A) is an absolute set

2.1. Soft Subset [6]: Consider two soft sets in U, (F, A) and $(\mathrm{G}, \quad \mathrm{B}) . \quad(\mathrm{F}, \mathrm{A}) \subseteq(\mathrm{G}, \mathrm{B})$ if $\mathrm{A} \subseteq \mathrm{B}$ and $\forall a_{i} \in A, F\left(a_{i}\right) \subseteq G\left(a_{i}\right)$

$(\mathrm{F}, \mathrm{A}) \subseteq(\mathrm{G}, \mathrm{B})$ and $(\mathrm{G}, \mathrm{B}) \subseteq{ }_{(\mathrm{F}, \mathrm{A})}$ then $(\mathrm{F}, \mathrm{A})=(\mathrm{G}, \mathrm{B})$

2.2. AND Product: The AND product of two soft sets $(\mathrm{F}, \mathrm{A})$ and $(\mathrm{G}, \mathrm{B})$ is denoted by $(\mathrm{F}, \mathrm{A}) \wedge(\mathrm{G}, \mathrm{B})$ such that $(\mathrm{F}, \mathrm{A}) \wedge$ $(\mathrm{G}, \mathrm{B})=\left(\mathrm{P}, \mathrm{A} \times{ }_{\mathrm{B}}\right)$ where $P\left(a_{i}, b_{j}\right)=F\left(a_{i}\right) \cap G\left(b_{j}\right)$ and $\left(a_{i}, b_{j}\right) \in A \times B$

2.3. OR Product: The OR product of two soft sets (F,A) and $(\mathrm{G}, \mathrm{B})$ is denoted by $(\mathrm{F}, \mathrm{A}) \vee(\mathrm{G}, \mathrm{B})$ such that $(\mathrm{F}, \mathrm{A}) \vee(\mathrm{G}, \mathrm{B})$ $=(\mathrm{P}, \mathrm{A} \times \mathrm{B})$ where $P\left(a_{i}, b_{j}\right)=F\left(a_{i}\right) \cup G\left(b_{j}\right)$ and $\left(a_{i}, b_{j}\right) \in A \times B$

2.4. Union[17]: The union of two soft sets $(F, A)$ and $(\mathrm{G}, \mathrm{B})$ is denoted by $(\mathrm{F}, \mathrm{A}) \widetilde{\cup}(\mathrm{G}, \mathrm{B})$ and if $(H, C)=(F, A) \widetilde{\cup}(G, B)$ then $c \in C=A \cup B$, also $\forall c \in C$

$$
H(c)=\left\{\begin{array}{cc}
F(c) & \text { if } c \in A-B \\
G(c) & \text { if } c \in B-A \\
F(c) \cup G(c) & \text { if } c \in A \cap B
\end{array}\right.
$$

2.5. Intersection[18]: The two most common types of Intersections for soft sets are:-
$F(e 4)=\{(v 1,[0.3,0.6]),(v 3,[0.1,0.4]),(v 4,[0.5,0.9]),(v 5,[0.4,0.7]$ )$,(\mathrm{v} 7,[0.1,0.3])\}$

$\mathrm{F}(\mathrm{e} 5)=\{(\mathrm{v} 3,[0,0.2]),(\mathrm{v} 6,[0.7,0.9]),(\mathrm{v} 7,[0.6,0.8])\}$

$\mathrm{F}(\mathrm{e} 6)=\{(\mathrm{v} 1,[0.3,0.7]),(\mathrm{v} 2,[0.5,0.9])\}$

$F(e 7)=\{(v 2,[0.1,0.3]),(v 3,[0,0.2]),(v 4,[0.1,0.4]),(v 6,[0.2,0.5])\}$ In matrix/ tabular form

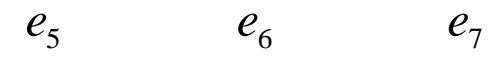

$\begin{array}{lll}0 & {[0.3,0.7] \quad 0}\end{array}$

$\begin{array}{lll}0 & {[0.5,0.9] \quad[0.1,0.3]}\end{array}$

$[0,0.2] \quad 0 \quad[0,0.2]$

0

0

$[0.1,0.4]$

0

0

0

$[0.7,0.9] \quad 0 \quad[0.2,0.5]$

$[0.6,0.8] \quad 0 \quad 0$

1. Restricted intersection of two soft sets $(F, A)$ and $(G, B)$ is denoted by $(F, A) \quad \tilde{\cap} \quad(G, B)$ and if $(H, C)=(F, A) \tilde{\cap}(G, B)$ then $e \in C=A \cap B$, also $\forall e \in C, H(e)=F(e) \cap G(e)$

2. Extended intersection of two soft sets $(F, A)$ and $(G, B)$ is denoted by $(F, A) \cap_{E} \quad(G, B)$ and if $(H, C)=(F, A) \cap_{E}(G, B)$ then $c \in C=A \cup B$, also $\forall c \in C$

$$
H(c)=\left\{\begin{array}{cc}
F(c) & \text { if } c \in A-B \\
G(c) & \text { if } c \in B-A \\
F(c) \cap G(c) & \text { if } c \in A \cap B
\end{array}\right.
$$

Note: All these functions apply to FSS and IVFSS in the same way.

\section{DATA IMPLEMENTATION AND OBSERVATION}

\subsection{Initial Data Set}

The following patient data was taken such that each values were diverse and hence enabled us to observe the correlation of the factors. A total of 30 patients of different age groups were taken having quite different PSA and PV figures. The PSA Density was calculated later on. Some of the patients, on which the steps performed in this study will be demonstrated, are as follows:-

\begin{tabular}{c|c|c|c|c}
\hline Patient & Age & $\begin{array}{c}\text { PSA } \\
(\mathbf{n g} / \mathrm{ml})\end{array}$ & $\begin{array}{c}\text { PV } \\
(\mathbf{c c})\end{array}$ & $\begin{array}{c}\text { PSA Density } \\
(\mathbf{n g} / \mathrm{ml} / \mathbf{c c})\end{array}$ \\
\hline $\mathbf{e}_{\mathbf{1}}$ & 52 & 3.68 & 46 & 0.08 \\
\hline $\mathbf{e}_{\mathbf{2}}$ & 56 & 4.416 & 39 & 0.096 \\
\hline
\end{tabular}


International Journal of Computer Applications (0975 - 8887)

Volume 177 - No. 38, February 2020

\begin{tabular}{|c|c|c|c|c|}
\hline $\mathbf{e}_{\mathbf{5}}$ & 65 & 6.426 & 63 & 0.102 \\
\hline $\mathbf{e}_{\mathbf{6}}$ & 63 & 9.24 & 77 & 0.12 \\
\hline $\mathbf{e}_{\mathbf{8}}$ & 49 & 11.4 & 57 & 0.20 \\
\hline $\mathbf{e}_{\mathbf{1 0}}$ & 63 & 20.8 & 65 & 0.32 \\
\hline $\mathbf{e}_{\mathbf{1 2}}$ & 76 & 10.66 & 82 & 0.13 \\
\hline $\mathbf{e}_{\mathbf{1 3}}$ & 80 & 22.91 & 79 & 0.29 \\
\hline $\mathbf{e}_{\mathbf{1 7}}$ & 49 & 10.83 & 57 & 0.19 \\
\hline $\mathbf{e}_{\mathbf{1 8}}$ & 59 & 7.68 & 64 & 0.12 \\
\hline $\mathbf{e}_{\mathbf{2 1}}$ & 40 & 6.5 & 50 & 0.13 \\
\hline
\end{tabular}

\begin{tabular}{|c|c|c|c|c|}
\hline $\mathbf{e}_{22}$ & 36 & 7.92 & 48 & 0.165 \\
\hline $\mathbf{e}_{25}$ & 50 & 7.02 & 78 & 0.09 \\
\hline $\mathbf{e}_{26}$ & 44 & 9.9 & 75 & 0.132 \\
\hline $\mathbf{e}_{30}$ & 47 & 9.617 & 59 & 0.163 \\
\hline
\end{tabular}

\subsection{Assigning Fuzzy Values}

In order to set up a standard for differentiating low from high, small from big and an adult from an elder, we convert the following values to assign each factor linguistic groups and for each linguistic group we will set fuzzy membership values.

\section{Age Groups}

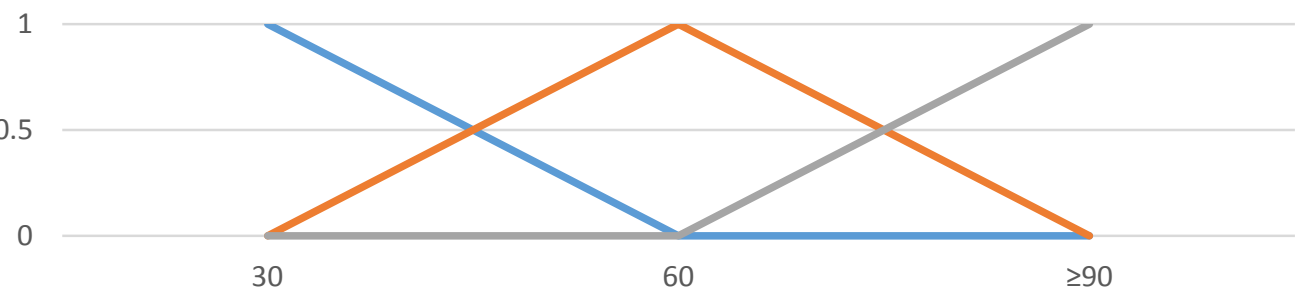

$\longrightarrow$ Adult-Middle(A-M) Middle-Old(M-O) $\longrightarrow$ Old-Elder (O-E)

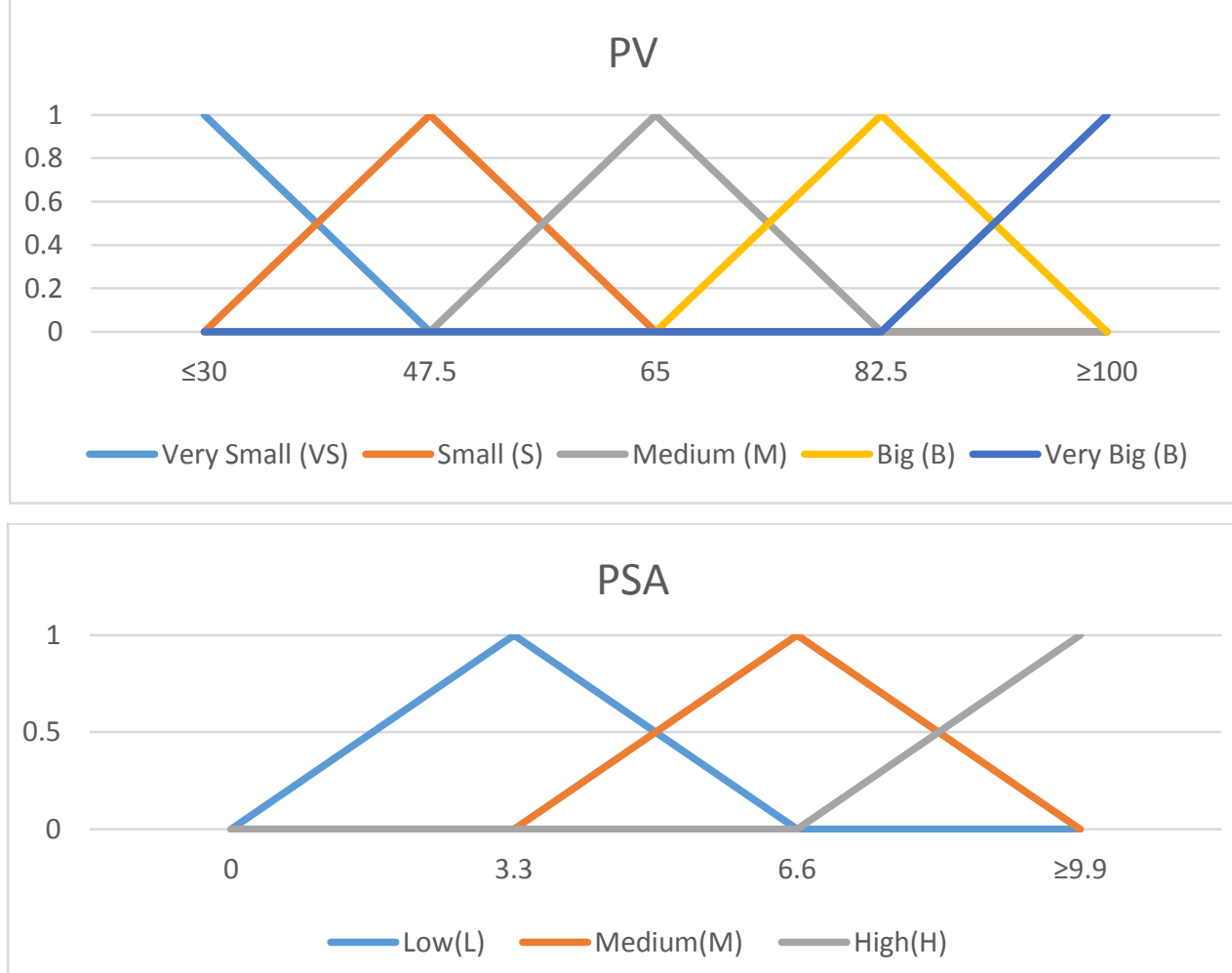






The functions determining each fuzzy value for are:-

$$
\begin{gathered}
\text { Age }(w)=\left\{\begin{array}{lc}
\alpha_{A-M} & w \leq 30 \\
\alpha_{M-O} & 30<w \leq 60 \\
\alpha_{O-E} & 60<w \leq 90 \text { or } w \geq 90
\end{array}\right. \\
P S A(x)=\left\{\begin{array}{lc}
\alpha_{L}(x) & 0<x \leq 3.3 \\
\alpha_{M}(x) & 3.3<x \leq 6.6 \\
\alpha_{H}(x) & 6.6<x \leq 9.9 \text { or } x \geq 9.9
\end{array}\right. \\
P V(y)=\left\{\begin{array}{lc}
\alpha_{V S}(y) & y \leq 30 \\
\alpha_{S}(y) & 30<y \leq 47.5 \\
\alpha_{M}(y) & 47.5<y \leq 65 \\
\alpha_{B}(y) & 65<y \leq 82.5 \\
\alpha_{V B}(y) & 82.5<y \leq 100 \text { or } y \geq 100
\end{array}\right.
\end{gathered}
$$

\begin{tabular}{|c|c|c|c|c|}
\hline $\begin{array}{c}\text { Patie } \\
\text { nt }\end{array}$ & Age & $\begin{array}{c}\text { PSA } \\
(\mathrm{ng} / \mathrm{ml})\end{array}$ & $\begin{array}{l}\text { PV } \\
\text { (cc) }\end{array}$ & $\begin{array}{c}\text { PSA } \\
\text { Density } \\
\text { (ng/ml/cc) }\end{array}$ \\
\hline $\mathbf{e}_{1}$ & $\begin{array}{c}0.27 \mathrm{M}, 0.7 \\
3 \mathrm{O}\end{array}$ & $\begin{array}{c}0.91 \mathrm{~L}, 0.09 \\
\mathrm{M}\end{array}$ & $\begin{array}{c}0.09 \mathrm{VS}, 0.9 \\
1 \mathrm{~S}\end{array}$ & $\begin{array}{c}0.67 \mathrm{~L}, 0.33 \\
\mathrm{M}\end{array}$ \\
\hline $\mathbf{e}_{2}$ & $\begin{array}{c}0.14 \mathrm{M}, 0.8 \\
6 \mathrm{O}\end{array}$ & $\begin{array}{c}0.73 \mathrm{~L}, 9.27 \\
\mathrm{M}\end{array}$ & $\begin{array}{c}0.49 \mathrm{VS}, 0.5 \\
1 \mathrm{~S}\end{array}$ & $0.4 \mathrm{~L}, 0.6 \mathrm{M}$ \\
\hline$e_{5}$ & $\begin{array}{c}0.72 \mathrm{O}, 0.2 \\
8 \mathrm{E}\end{array}$ & $\begin{array}{c}0.06 \mathrm{~L}, 0.94 \\
\mathrm{M}\end{array}$ & $\begin{array}{c}0.12 \mathrm{~S}, 0.88 \\
\mathrm{M}\end{array}$ & $0.3 \mathrm{~L}, 0.7 \mathrm{M}$ \\
\hline$e_{6}$ & $0.9 \mathrm{O}, 0.1 \mathrm{E}$ & $0.2 \mathrm{M}, 0.8 \mathrm{H}$ & $\begin{array}{c}0.32 \mathrm{M}, 0.6 \\
8 \mathrm{~B}\end{array}$ & $1 \mathrm{M}$ \\
\hline$e_{8}$ & $\begin{array}{c}0.37 \mathrm{M}, 0.6 \\
3 \mathrm{O}\end{array}$ & $1 \mathrm{H}$ & $\begin{array}{c}0.46 \mathrm{~S}, 0.54 \\
\mathrm{M}\end{array}$ & $1 \mathrm{H}$ \\
\hline $\mathbf{e}_{10}$ & $0.9 \mathrm{O}, 0.1 \mathrm{E}$ & $1 \mathrm{H}$ & $1 \mathrm{M}$ & $1 \mathrm{H}$ \\
\hline $\mathbf{e}_{12}$ & $\begin{array}{c}0.47 \mathrm{O}, 0.5 \\
3 \mathrm{E}\end{array}$ & $1 \mathrm{H}$ & $\begin{array}{c}0.03 \mathrm{M}, 0.9 \\
7 \mathrm{~B}\end{array}$ & $\begin{array}{c}0.84 \mathrm{M}, 0.1 \\
6 \mathrm{H}\end{array}$ \\
\hline $\mathbf{e}_{13}$ & $\begin{array}{c}0.340,0.6 \\
6 \mathrm{E}\end{array}$ & $1 \mathrm{H}$ & $0.2 \mathrm{M}, 0.8 \mathrm{~B}$ & $1 \mathrm{H}$ \\
\hline $\mathbf{e}_{17}$ & $\begin{array}{c}0.37 \mathrm{M}, 0.6 \\
3 \mathrm{O}\end{array}$ & $1 \mathrm{H}$ & $\begin{array}{c}0.54 \mathrm{~S}, 0.46 \\
\text { B }\end{array}$ & $1 \mathrm{H}$ \\
\hline
\end{tabular}

The table displaying the fuzzy membership values for the FSSs developed in this process is as follows:-

\begin{tabular}{c|c|c|c|c|}
\hline $\mathbf{e}_{18}$ & $0.04 \mathrm{M}, 0.9$ & $0.67 \mathrm{M}, 0.3$ & $0.06 \mathrm{~S}, 0.94$ & $1 \mathrm{M}$ \\
& $6 \mathrm{O}$ & $3 \mathrm{H}$ & $\mathrm{M}$ & \\
\hline $\mathbf{e}_{21}$ & $0.67 \mathrm{M}, 0.3$ & $0.04 \mathrm{~L}, 0.96$ & $0.86 \mathrm{~S}, 0.14$ & $0.84 \mathrm{M}, 0.1$ \\
& $3 \mathrm{O}$ & $\mathrm{M}$ & $\mathrm{M}$ & $6 \mathrm{H}$ \\
\hline $\mathbf{e}_{22}$ & $0.8 \mathrm{M}, 0.2 \mathrm{O}$ & $0.6 \mathrm{M}, 0.4 \mathrm{H}$ & $0.97 \mathrm{~S}, 0.03$ & $0.15 \mathrm{M}, 0.7$ \\
\hline $\mathbf{e}_{25}$ & $0.34 \mathrm{M}, 0.6$ & $0.87 \mathrm{M}, 0.1$ & $0.26 \mathrm{M}, 0.7$ & $0.5 \mathrm{~L}, 0.5 \mathrm{M}$ \\
& $6 \mathrm{O}$ & $3 \mathrm{H}$ & $4 \mathrm{~B}$ & \\
\hline $\mathbf{e}_{26}$ & $0.54 \mathrm{M}, 0.4$ & $1 \mathrm{H}$ & $0.43 \mathrm{M}, 0.5$ & $0.8 \mathrm{M}, 0.2 \mathrm{H}$ \\
& $6 \mathrm{O}$ & & $7 \mathrm{~B}$ & \\
\hline $\mathbf{e}_{30}$ & $0.44 \mathrm{M}, 0.5$ & $0.09 \mathrm{M}, 0.9$ & $0.34 \mathrm{M}, 0.6$ & $0.28 \mathrm{M}, 0.7$ \\
& $6 \mathrm{O}$ & $1 \mathrm{H}$ & $6 \mathrm{~S}$ & $2 \mathrm{H}$ \\
& & & & \\
\hline
\end{tabular}

These fuzzy membership ratios help us determine the patients' age group, PSA levels, PV and PSA density, without needing to consult the standard PSA, PV or PSA density scale. But we have not yet been enabled to draw a conclusion from this information. To correlate the following factors, we need to shorten the numerical values whilst still addressing each unique value within a linguistic group.

\subsection{Implementing the linguistic variables into IVFSS}

We convert each linguistic variable in to a Interval Value to shorten the data sets. The general form of the IVFSSs will be as follows:-

$$
\begin{aligned}
& F([0,1])_{\text {Age }}=\left\{\left(e_{A-M}=[0,0.33]\right),\left(e_{M-O}=[0.34,0.66]\right),\left(e_{0-E}=[0.67,1]\right)\right\} \\
& F([0,1])_{P S A}=\left\{\left(e_{L}=[0,0.33]\right),\left(e_{M}=[0.34,0.66]\right),\left(e_{H}=[0.67,1]\right)\right\} \\
& F([0,1])_{P V}=\left\{\begin{array}{l}
\left(e_{V S}=[0,0.2]\right),\left(e_{S}=[0.21,0.4]\right),\left(e_{M}=[0.41,0.6]\right), \\
\left(e_{B}=[0.61,0.8]\right),\left(e_{V B}=[0.81,1]\right)
\end{array}\right\} \\
& F([0,1])_{P S A \text { Density }}=\left\{\left(e_{L}=[0,0.33]\right),\left(e_{M}=[0.34,0.66]\right),\left(e_{H}=[0.67,1]\right)\right\}
\end{aligned}
$$

The IVFSSs formed on the basis of this data were:-

For age:-

$$
\begin{aligned}
& F([0.34,0.66])_{\text {Age }}=\left\{e_{16}, e_{21}, e_{22}, e_{23}, e_{24}, e_{26}, e_{27} e_{29}\right\} \\
& F([0.67,1])_{A g e}=\left\{\begin{array}{l}
e_{1}, e_{2}, e_{3}, e_{4}, e_{5}, e_{6}, e_{7}, e_{8}, e_{9}, e_{10}, e_{11}, e_{12}, e_{13}, \\
e_{14}, e_{15}, e_{17}, e_{18}, e_{19}, e_{20}, e_{25}, e_{28}, e_{30}
\end{array}\right\}
\end{aligned}
$$

For PSA:-

$$
F([0,0.33])_{P S A}=\left\{e_{1}, e_{2}, e_{3}\right\}
$$




$$
\begin{aligned}
& F([0.34,0.66])_{P S A}=\left\{e_{4}, e_{5}, e_{18}, e_{21}, e_{21}, e_{22}, e_{24}, e_{25}\right\} \\
& F([0.34,0.66])_{P S A}=\left\{e_{4}, e_{5}, e_{18}, e_{21}, e_{21}, e_{22}, e_{24}, e_{25}\right\} \\
& F([0.67,1])_{P S A}=\left\{\begin{array}{l}
e_{6}, e_{7}, e_{8}, e_{9}, e_{10}, e_{11}, e_{12}, e_{13}, e_{14}, e_{15}, e_{16}, e_{17} \\
e_{19}, e_{20}, e_{23}, e_{26}, e_{27}, e_{28}, e_{29}, e_{30}
\end{array}\right\}
\end{aligned}
$$

For PV:-

$F([0.21,0.4])_{P V}=\left\{e_{1}, e_{2}, e_{3}, e_{7}, e_{16}, e_{17}, e_{21}, e_{22}, e_{23}, e_{24}, e_{30}\right\}$ $F([0.41,0.6])_{P V}=\left\{e_{4}, e_{5}, e_{8}, e_{9}, e_{10}, e_{11}, e_{14}, e_{15}, e_{18}, e_{19}, e_{20}, e_{27}, e_{29}\right\}$ $F([0.61,0.8])_{P V}=\left\{e_{6}, e_{12}, e_{13}, e_{25}, e_{26}, e_{28}\right\}$

For PSA Density:-

$F([0.34,0.66])_{A g e} \wedge F([0.34,0.66])_{P S A} \wedge F([0.21,0.4])_{P V} \wedge F([0.34,0.66])_{P S A \text { Density }}$ $=\left\{e_{21}, e_{24}\right\}$

Product 2:

$F([0.34,0.66])_{A g e} \wedge F([0.34,0.66])_{P S A} \wedge F([0.21,0.4])_{P V} \wedge F([0.67,1])_{P S A \text { Density }}$ $=\left\{e_{22}\right\}$

Product 3:

$F([0.34,0.66])_{A g e} \wedge F([0.67,1])_{P S A} \wedge F([0.21,0.4])_{P V} \wedge F([0.67,1])_{P S A \text { Density }}$ $=\left\{e_{16}, e_{23}\right\}$

Product 4:

$F([0.34,0.66])_{A g e} \wedge F([0.34,0.66])_{P S A} \wedge F([0.21,0.4])_{P V} \wedge F([0.34,0.66])_{P S A \text { Density }}$ $=\left\{e_{21}, e_{24}\right\}$

Product 5:

$F([0.34,0.66])_{A g e} \wedge F([0.67,1])_{P S A} \wedge F([0.61,0.8])_{P V} \wedge F([0.34,0.66])_{P S A \text { Density }}$ $=\left\{e_{26}\right\}$

Product 6:

$F([0.67,1])_{A g e} \wedge F([0,0.33])_{P S A} \wedge F([0.21,0.4])_{P V} \wedge F([0,0.33])_{P S A \text { Density }}$ $=\left\{e_{1}, e_{3}\right\}$

Product 7:

$F([0.67,1])_{A g e} \wedge F([0,0.33])_{P S A} \wedge F([0.21,0.4])_{P V} \wedge F([0.34,0.66])_{P S A \text { Density }}$ $=\left\{e_{2}\right\}$

Product 8:

$F([0.67,1])_{A g e} \wedge F([0.34,0.66])_{P S A} \wedge F([0.41,0.6])_{P V} \wedge F([0.34,0.66])_{P S A \text { Density }}$ $=\left\{e_{4}, e_{5}, e_{18}\right\}$

Product 9:

$F([0.67,1])_{A g e} \wedge F([0.34,0.66])_{P S A} \wedge F([0.61,0.8])_{P V} \wedge F([0,0.33])_{P S A \text { Density }}$ $=\left\{e_{25}\right\}$

Product 10:

$$
\begin{aligned}
& F([0,0.33])_{\text {PSADensity }}=\left\{e_{1}, e_{3}, e_{5}\right\} \\
& F([0.34,0.66])_{\text {PSADensity }}=\left\{e_{2}, e_{4}, e_{5}, e_{6}, e_{12}, e_{18}, e_{21}, e_{24}, e_{26}, e_{27}\right\} \\
& F([0.67,1])_{\text {PSADensity }}=\left\{\begin{array}{l}
e_{7}, e_{8}, e_{9}, e_{10}, e_{11}, e_{13}, e_{14}, e_{15}, e_{16}, e_{17}, e_{19} \\
e_{20}, e_{22} e_{23}, e_{28}, e_{29}, e_{30}
\end{array}\right\}
\end{aligned}
$$

the have dwindled the FSS to a few IVFSSs, we can and correlate the patients with the factors as a consequence. Some of the AND products were as follows:-

Product 1: 


$$
\begin{aligned}
& F([0.67,1])_{A g e} \wedge F([0.67,1])_{P S A} \wedge F([0.21,0.4])_{P V} \wedge F([0.67,1])_{P S A \text { Density }} \\
& =\left\{e_{7}, e_{17}, e_{30}\right\}
\end{aligned}
$$

Product 11:

$$
\begin{aligned}
& F([0.67,1])_{A g e} \wedge F([0.67,1])_{P S A} \wedge F([0.41,0.6])_{P V} \wedge F([0.67,1])_{P S A \text { Density }} \\
& =\left\{e_{8}, e_{9}, e_{10}, e_{11}, e_{14}, e_{15}, e_{19}, e_{20}\right\}
\end{aligned}
$$

Product 12:

$$
\begin{aligned}
& F([0.67,1])_{A g e} \wedge F([0.67,1])_{P S A} \wedge F([0.61,0.8])_{P V} \wedge F([0.34,0.66])_{P S A \text { Density }} \\
& =\left\{e_{6}, e_{12}\right\}
\end{aligned}
$$

Product 13:

$$
\begin{aligned}
& F([0.67,1])_{\text {Age }} \wedge F([0.67,1])_{P S A} \wedge F([0.61,0.8])_{P V} \wedge F([0.67,1])_{P S A \text { Density }} \\
& =\left\{e_{13}\right\}
\end{aligned}
$$

\subsection{Analysis}

Observing these products and already diagnosed prostate cancer patients, following results can be drawn:-

1- Few products gave empty sets and, although, have not been included here, are crucial for making our algorithm to further developing the code for the risk calculator. To improvise, consider that a 40 year old man cannot have a PV above 75 and unless his PSA is really high. Similarly no person can have alarming PSA and have normal PSA density.

2- PSA density is more suitable to determine potential cancer risk than PSA alone since the normal PSA values and the correlation of PSA to PV are subject to age and, however minimally, to race as well[19-24].

3- PV can be larger than normal in a patient and yet the patient may not have higher values of PSA to justify that. This indicates possibility of BPH (Benign Prostate Hyperplasia).

4- The impact factor of each attribute can be divided as follows

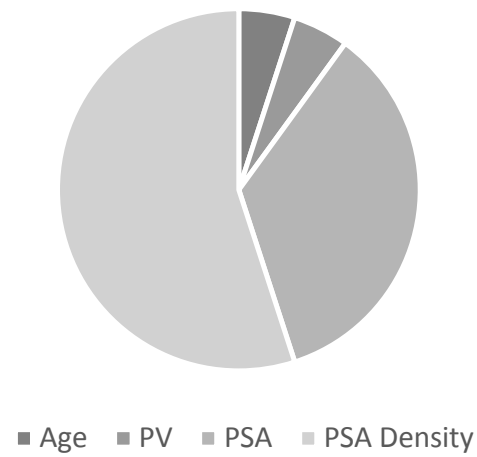

\subsection{Algorithm and Programming}

From the results drawn above, it is now convenient to create an algorithm on the basis of which our program will function.

1- Input Age,PSA, PV

2- Density=PSA/PV

3- Age_percent $=($ Age $/ 90) \times 5$

4- If Age_percent $>5$, then Age_percent $=5$

5- age_val=(Age/90)
6- If age_val $>1$, then age_val $=1$

7- If age_val $\leq 0.33$, then age_group=adult_middle

8 - Else If $0.33<$ age_val $\leq 0.66$, then age_group=middle_old

9- Else If $0.66<$ age val $\leq 1$, then age group $=$ old elder

10- If age_group=adult_middle, then PSA_percent $=(\mathrm{PSA} / 3.5) \times 30$

11- Else If age_group=middle_old, then PSA_percent $=(\mathrm{PSA} / 6.5) \times 30$

12- Else If age_group $=$ old_elder, then PSA_percent $=(\mathrm{PSA} / 9.5) \times 30$

13- If PSA_percent $>30$, then PSA_percent $=30$

14- PV_percent $=(P V / 100) \times 5$

15- If PV_percent $>5$ then PV_percent=5.

16- Density_percent=(Density/0.18) $\times 55$

17- Print "The Prostate cancer risk percentage for the potential patient

is $=$ " Age_percent+PSA_percent+PV_percent+Density_percent.

18-If PV_percent $>2.5$ and Density_percent $<25$ then Print "The patient may have BPH"

Based on the following algorithm we wrote the program in Python. However, the code is quite simplistic and can be written in any object oriented programming language.

\section{CONCLUSION}

This study utilizes FSST and IVFSST to acknowledge the impact factor of each attribute that determines the state of prostate within a patient. The FSS gives us membership values for each attribute but using the FSSs would be inconvenient in case of larger data sets and deducing an algorithm with that information alone would be difficult. Thus we converted the linguistic variables into interval values and observed where the patients lie with respect to their attributes from fuzzy membership degrees. The algorithm is later on derived with the help of results observed by taking AND product of the said interval values of the attributes. A program coded on this algorithm can help a doctor if prostate cancer biopsy is necessary for a patient. If the cancer risk is above $50 \%$, the patient should get prostate cancer biopsy. Based on our research, we were not only able to pinpoint the patients in this 
data set who had contracted prostate cancer but also determine potential BPH patients. There is another attribute PSA Velocity which can be looked at, to determine prostate cancer risk, but that is inconvenient to implement in this study without the knowledge of PSA values of a patient in the past year.

\section{REFERENCES}

[1] Zadeh L.A.:Fuzzy sets.Inf. Control 8, 338-353 (1965)

[2] Molodstov D.: Soft set theory-first results. Comput.Math.Appl.,37(4-5), 19-31 (1999)

[3] Maji P.K., Roy A.R., Biswas R.: Fuzzy soft sets.J.Fuzzy Math., 9(3), 589-602 (2001)

[4] Ali M.I., Shabir M., Comments on De Morgan's law in fuzzy soft sets. J.Fuzzy Math., 18(3), 679-686 (2010)

[5] Feng F., Jun.Y.B., Liu.X.Y., Li L.F.: An adjustable approach to fuzzy soft set based decision making. J.Comput.Appl.Math., 234, 10-20 (2010)

[6] Feng F., Li C., Davvaz B., Ali M.I.: Soft sets combined with fuzzy sets and rough sets. Soft Comput., 14, 899-911 (2010)

[7] Yang X.B., Lin T.Y., Yang J.Y., Li Y., and Yu D.: Combination of interval-valued fuzzy set and soft set. Computers and Mathematics with Applications ,58(3), 521-527 (2009)

[8] Catolona W.J., Partin A.W., Slawin K.M., Brawer M.K., Flanigan R.C., Patel. A. et al.: Use of the percentage free prostage specific antigen to enhance differentiation of prostate cancer from benign prostatic disease: a prospective multicenter clinical trial,.JAMA, Am. Med. Assoc. 279, 1542-1547 (1998)

[9] Egawa S., Soh S. Ohori M., Uchida T., Gohji K., Fujii A. et al: The ratio of free to total serum prostate specific antigen and its use in differential diagnosis of prostate carcinoma in Japan. Cancer(Online) 79, 90-98 (1997)

[10] Van Cangh P.J., De Nayer P., De Vischer L., Sauvage P., Tombal B., Lorge F. et al.: Free to total prostate specific antigen (PSA) ratio is superior to total PSA in differentiating benign prostate hypertrophy from prostate cancer. Prostate (Online) 29, 30-34 (1996)

[11] Nguyen.H.P., Kreinovich V.: Fuzzy Logic and its application in medicine. Int.J.Med. 62, 165-173 (2001)

[12] Metlin C., Lee F., Drago J.: The American cancer society national prostate cancer detection project. Findings on the detection of early prostate cancer in 2425 men. Cancer (Online) 67, 2949-2958 (1991)

[13] Seker H., Odetayo M., Petrovic D., Naguib R.N.G..,: A fuzzy logic based method for prognostic decision making in breast cancer and prostate cancers, IEEE Trans. Inf. Technol. Biomed., 7, 114-122 (2003)

[14] Saritas I., Allahverdi N., Sert U.: A fuzzy expert system design for diagnosis of prostate cancer. International on Computer Systems and Technologies - CompSysTech 2003 (2003)

[15] Keles A., Hasiloglu A.S., Aksoy Y., Neuro-fuzzy classification of prostate cancer using NEFCLASS-J Comput. Comput. Biol. Med., 37, 1617-1628(2007)
[16] Saritas I., Ozkan I.A., Sert U.: Prognosis of prostate cancer by artificial neural networks. Expert Syst. Appl. 37, 6646-6650 (2010)

[17] Maji P.K., Biswas R., Roy A.R.: Soft set theory. Comput. Math. Appl., 45, 555-562 (2003)

[18] Ali M.I., Feng F., Liu X., Min W.K., Shabir M.: On some new operations in soft set theory. Comput. Math. Appl., 57, 1547-1553 (2009)

[19] Mosli H., Abdel-Meguid T.: The relationship between prostate volume, prostate specific antigen and age in Saudi men with benign prostatic conditions. Afr. J. Urol. 16, 117-123 (2010)

[20] Vesely S., Knutson T., Damber J.E., Dicuio M., Dahlstrand C.: Relationship between age, prostate volume, prostate specific antigen, symptom score and uroflowmetry in men with lower urinary tract symptoms. Scand. J. Urol. Nephrol 37, 322-328 (2003)

[21] Roehborn C.G., Boyle P., Gould A.L., Waldstreicher J.: Serum prostate specific antigen as a predictor of prostate volume in men with benign prostate hyperplasia. Urology 53, 581-589 (1999)

[22] Gupta A., Aragaki C., Gotoh M., Masumori N., Ohshima S. Tsukamoto $\mathrm{T}$. et al. Relationship between prostate specific antigen and indexes of prostate volume in Japanese men. J.Urol. 173, 503-506 (2005)

[23] Chang Y.L., Lin A.T., Chen K.K., Chang Y.H., Wu H.H., Kuo J.Y. et al. Correlation between serum prostate specific antigen and prostate volume in Taiwanese men with biopsy proven benign prostatic hyperplasia. J. Urol. 176, 196-199(2006)

[24] Mochtar C.A. Kiemeney L.A., Van Riemsdijk M.M., Barnett G.S., Laguna M.P., Debruyne F.M. et al.: Prostate specific antigen as an estimator of prostate volume in the management of patients with symptomatic benign prostatic hyperplasia. Eur. Urol. 44, 695-700(2003)

[25] Jafar.N.M, Saqlain.M, Saeed.M, Abbas.Q (2020), Application of Soft-Set Relations and Soft Matrices in Medical Diagnosis using Sanchez's Approach , International Journal of Computer Applications, 177(32): $7-11$.

[26] Jafar.N.M, Muniba.K, Saeed.A, Abbas.S, Bibi.I (2019), Application of Sanchez's Approach to Disease Identification Using Trapezoidal Fuzzy Numbers, International Journal of Latest Engineering Research and Applications, 4(9):51-57

[27] Jafar.N.M, Faizullah, Shabbir.S, Alvi.F.M.S, Shaheen.L (2019), Intuitionistic Fuzzy Soft Matrices, Compliments and Their Relations with Comprehensive Study of Medical Diagnosis, International Journal of Latest Engineering Research and Applications, 5(1): 23-30.

[28] Riaz.M, Saeed.M, Saqlain.M, Jafar.N (2019), Impact of Water Hardness in Instinctive Laundry System Based on Fuzzy Logic Controller, Punjab University Journal of Mathematics, 51(4):73-84

[29] Saeed, M., Zulqarnain, M. and Dayan, F. (2018). TOPSIS analysis for the prediction of diabetes based on general characteristics of humans. Int. J. of Pharm. Sci. and Research. 9: 2932-2939 
[30] Saqlain.M.Jafar.N, Riffat.A (2018), Smart Phone Selection by Consumers' In Pakistan: FMCGDM Fuzzy Multiple Criteria Group Decision Making Approach, Gomal University Journal of Research, 34 (1): 27-31. 31.

[31] Saqlain.M, Jafar.N, Hamid.R,Shahzad.A. (2019), Prediction of Cricket World Cup 2019 by TOPSIS Technique of MCDM-A Mathematical Analysis, International Journal of Scientific \& Engineering Research, 10(2): 789-792
[32] Saqlain.M, Naz.K, Ghaffar.K, Jafar.N.M (2019), Fuzzy Logic Controller: The Impact of Water $\mathrm{pH}$ on Detergents, Scientific Inquiry of Review 3(3):16-29.

[33] Saqlain M, Saeed M, Ahmad M. R, Smarandache F, (2019), Generalization of TOPSIS for Neutrosophic Hypersoft set using Accuracy Function and its Application, Neutrosophic Sets and Systems (NSS), 27: 131-137. 